Ann. Geophys. Discuss., https://doi.org/10.5194/angeo-2019-41

Manuscript under review for journal Ann. Geophys.

Discussion started: 9 May 2019

(c) Author(s) 2019. CC BY 4.0 License.

\title{
1 Long Range Plasma Momentum Coupling by High Voltage Static Electric field 2 and Deep Space Exploration
}

\author{
3 Kokwei Chew ${ }^{1}$, Xinyu Zhou ${ }^{1}$, and Yian Lei ${ }^{1}$ \\ $4 \quad{ }^{1}$ School of Physics, Peking University, Beijing 100871, China \\ Correspondence to: Yian Lei (yalei@pku.edu.cn)
}

Abstract. Space exploration has been long constrained by the efficiency and capability of modern chemical rocket. Propellantless propulsion has been proposed as a solution to expand the boundary of space exploration. In this paper, we examine the possibilities of a propellantless propulsion scheme through the interaction between the spacecraft and ambient plasma. The spacecraft is charged to high electric potential by constantly shooting electrons away. The high voltage spacecraft will deplete the surrounding electrons, thus interact with a wide range of the background plasma (solar wind) and thus effectively extract momentum from the plasma. By taking advantage of the exploitable ambient plasma, a spacecraft can reach very high speed, thus considerably reducing the travel time. The scheme is also applicable for braking, which is helpful in the exploration of inner planets like Venus and Mercury, and the stopping at the destination planets or stars.

\section{Introduction}

Space exploration is the next step of human development. The main difficulty experienced in space travel is the long distance across planets, stars, or galaxies, and the low limitation of fuel. The average distance between planets in solar system is about 5.5 Astronomical units (AU) or 800 million km away, and the closest star system, the Alpha Centauri is 4.37 light-years $(4.1 \times$ $10^{16} \mathrm{~m}$ ) away from solar system. Contemporary spacecraft can hardly reach a speed of $40 \mathrm{kms}^{-1}$, with the help of multiple slingshot acceleration of the planets. The speed is far too low, considering the travelling to the Alpha Centauri will takes longer than the entire human history.

Propellantless propulsion is a scheme proposed to overcome the problems of limited fuels carried by chemical rockets as well as ion thrusters. To date, various kinds of propellantless schemes such as electric sail and solar sail has been proposed as an alternative to chemical rockets for interplanetary or interstellar space travelling. In this paper, we propose a propellantless propulsion scheme by coupling the spacecraft with the ambient plasma to extract its momentum. The spacecraft is charged to high electric potential by constantly shooting electrons far away. The high voltage spacecraft will interact with a wide range of the background plasma (solar wind) in momentum and gain thrust. Comparing with the electric sail proposed by P. Janhunen (2007, 2014), the effective radius of the spacecraft can be held within $100 \mathrm{~m}$ to provide better control and manoeuvring.

The key challenge in the engineering for this scheme is maintaining high potential of the spacecraft. The spacecraft has to accelerate and shot electrons far away to remain high potential and couple with ambient plasma. As electron is the 
Ann. Geophys. Discuss., https://doi.org/10.5194/angeo-2019-41

Manuscript under review for journal Ann. Geophys.

Discussion started: 9 May 2019

(c) Author(s) 2019. CC BY 4.0 License.

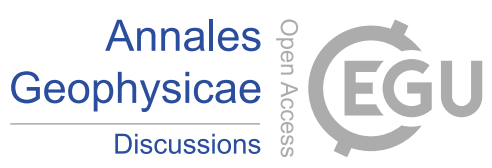

(c) (i)

magnets, electron acceleration is the easiest and most durable in engineering. Conventional non-superconducting electron injectors (Bluem et al. 2004) can accelerate electrons to $5 \mathrm{MeV}$ with a compact size, and operate continuously at a maximum current of 1 A. These two parameters set an upper limit on the potential and current of our scheme.

The feasibility of maintaining high potential also limited by the field ion evaporation effect or field evaporation (FEV) (Zurlev et al. 2003). FEV is the removal of surface atom from its own lattice structure by induced electric field. The whole process is rather complicated and not well studied yet. In general, when the electric field of a surface exceeded certain threshold, called $E^{e}$, there will be significant increase in the FEV process and deplete the structure. A theoretical estimation given by Dinko N. Zurlev (Forbes, 2006) showed that the $E^{e}$ for most metals is at the order of $10 \mathrm{Vnm}^{-1}$. For our spacecraft design with an effective radius of $100 \mathrm{~m}$, the threshold potential is at the order of $10^{12} \mathrm{~V}$, which is 6 orders of magnitude beyond our limit and thus should not be a problem.

\section{Concept}

The spacecraft interact with the ambient plasma through static electric field. Comparing with the electric sail design as proposed by P. Janhunen, our concept consists of a spacecraft located at the centre and linked to several positively charged lightweight balls by conducting tethers. The distance from spacecraft to the balls could be a few hundreds of meters. The spherical shape of balls is designed to reduce the FEV effect, and the distance of the balls is to effectively lower the potential of the structure, hence lower the energy of the ejected electron beam and improve energy efficiency. Due to electric static repulsion; all the balls will distributed uniform around the spacecraft. When deployed for operation, the electron gun is pointed to the opposite or perpendicular direction of the solar wind for ejecting the electrons away from the spacecraft.

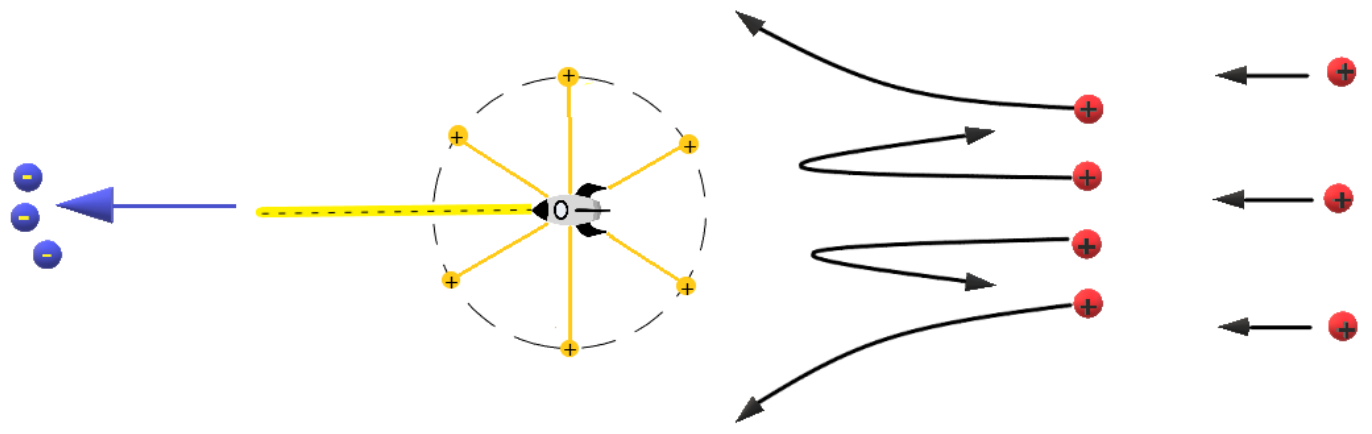

Figure 1: plasma wind coming from the right side being deflection by the electrostatic field of the spacecraft. The whole structure is consisted of a main unit at the center and connected to charged balls attached to the main unit.

For the sake of simplicity in simulation, the whole structure is treated as a sphere with the distance from spacecraft to the balls as the radius of the imaginary sphere. The sphere is charged to a surface potential of $V_{0}$, A stream of plasma (solar wind) moving from the positive $\mathrm{x}$ direction. We consider only 1 species of ion, the protons. The deflection of protons from its trajectories provides the momentum. The loss in $\mathrm{x}$-direction momentum of protons is transferred to the spacecraft. To calculate 
Ann. Geophys. Discuss., https://doi.org/10.5194/angeo-2019-41

Manuscript under review for journal Ann. Geophys.

Discussion started: 9 May 2019

(c) Author(s) 2019. CC BY 4.0 License.

the trajectory and hence the momentum, the potential generated by the sphere need to be addressed. For theoretical estimation, we consider the potential to be a central force and takes the forms of either Coulomb potential or Debye potential.

60 Where $r_{0}$ is the radius of the sphere and $V_{0}$ is the potential at the surface of the sphere and the potential at far point is zero.

61 Due to the presence of ambient plasma, shielding occurred and the potential dropped by and exponential factor. The effective

62 potential is:

$63 V(r)=\frac{V_{0} r_{0}}{r} e^{-\frac{r-r_{0}}{\lambda_{D}}}$,

64 Where $\lambda_{D}$ is the effective Debye length, $\lambda_{D}=\sqrt{\varepsilon_{0}\left(V_{0}-V_{1}\right) / n_{0} e}$ (Janhunen et al. 2016). $V_{1}$ is the kinetic energy of the 65 incoming proton.

We can estimate thrust by solving the equation of motion of the proton in a Coulomb potential. The deflection or scattering of protons, $\theta$ from its original direction is calculated from:

$68 b=\frac{V_{0} r_{0} e}{2 E_{k}} \cot \frac{\theta}{2}$,

Where $b$ is the impact parameter, $E_{k}$ is the kinetic energy of protons in solar wind. In this case, we set $\theta$ to 90 degree, the impact parameter $b$ represent how far the electric field can affect and repel the protons from solar wind. Assuming $V_{0}=1 \mathrm{MV}$, $r_{0}=100 \mathrm{~m}, E_{k}=1.5 \mathrm{keV}$, we can have the impact parameter $b=33 \mathrm{~km}$ and thus an effective scattering cross section of $3400 \mathrm{~km}^{2}$. The solar wind pressure at $1 \mathrm{AU}$ from the Sun is $2 \mathrm{nPa}$ and the spacecraft is estimated to acquire $6.8 \mathrm{~N}$ of thrust at the Earth's orbit.

In reality, the potential can never be a symmetric Coulomb potential due to the presence of plasma. The next part of this paper will provide simulation result for the actual thrust obtained by the spacecraft.

\section{1D Simulation}

In 1D simulation, the system will reach a static condition after the spacecraft is charged. The particles (ions) interact with the 78 charged spacecraft and eventually the potential around the spacecraft takes the form as described by equation 2 . The deflection of protons from its own trajectory contribute the x-direction momentum to the spacecraft. As shown in figure 2, the spacecraft is located at the origin. The ions is coming from the $+x$ direction and assumed to have initial velocity in the $x$-direction. The motion of the particle calculated by Runge-Kutta method with adaptive time-steps for error control. 
Ann. Geophys. Discuss., https://doi.org/10.5194/angeo-2019-41

Manuscript under review for journal Ann. Geophys.

Discussion started: 9 May 2019

(c) Author(s) 2019. CC BY 4.0 License.

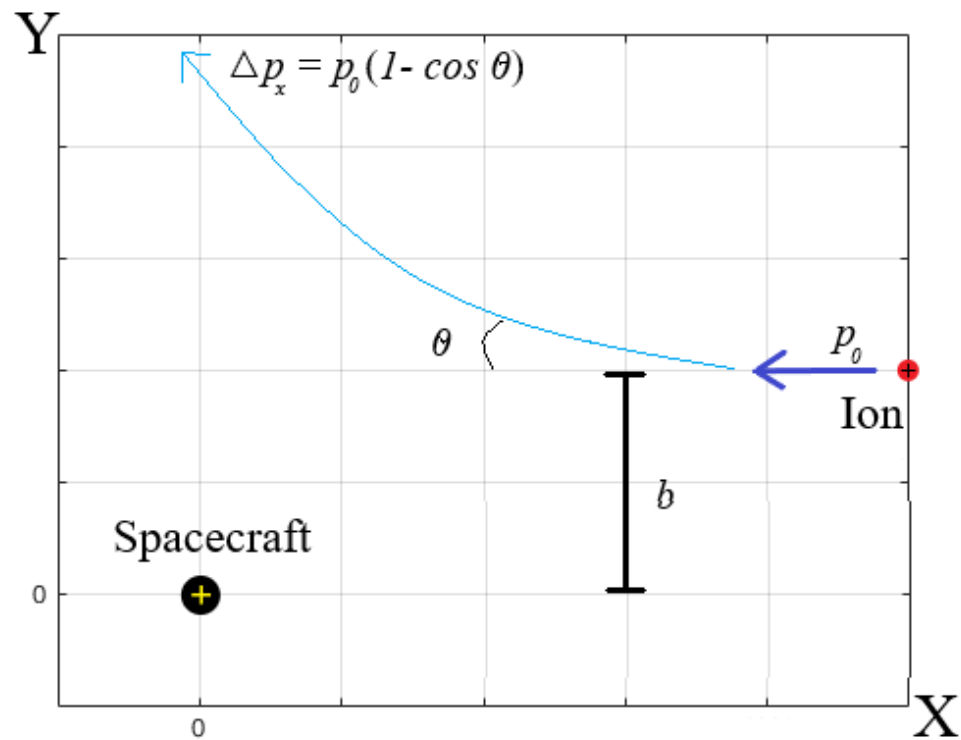

Figure 2: The spacecraft located at the origin and the ions from solar wind coming from the positive $x$ direction.

The model for 1D simulation is rather simple. It provides the relation between the thrust generated and key parameters of surface as well as solar wind density.

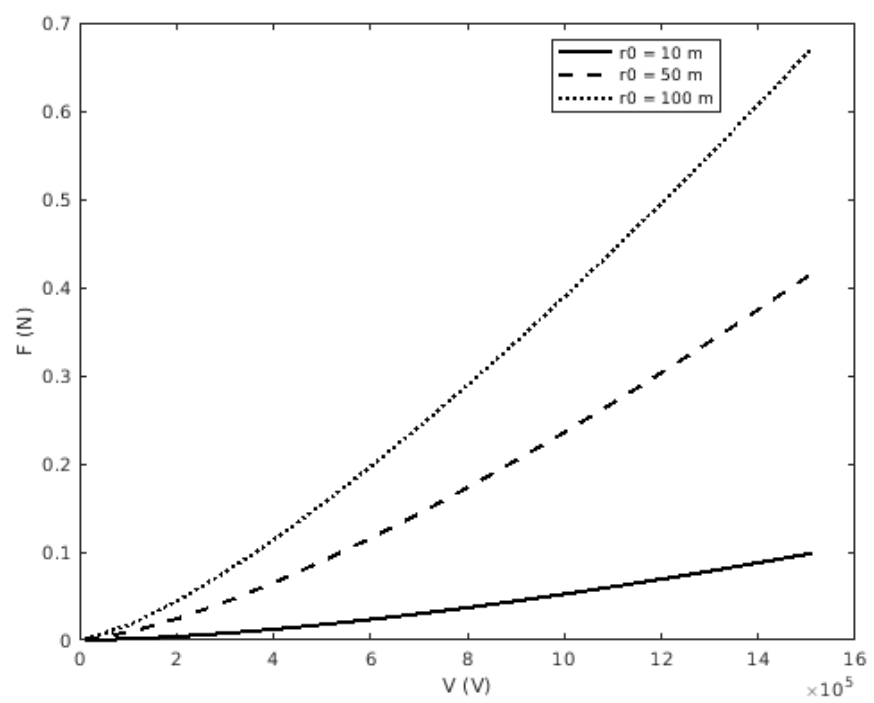

Figure 3(a): The relation between thrust $F$ and the surface potential of the sphere $V_{0}$. Dotted line: $r_{0}=100 \mathrm{~m}$, dashed line: $r_{0}=$ $50 \mathrm{~m}$, solid line: $r_{0}=10 \mathrm{~m}$. Plasma density $n_{0}=1.2 \times 10^{6} \mathrm{~m}^{-3}$. 
Ann. Geophys. Discuss., https://doi.org/10.5194/angeo-2019-41

Manuscript under review for journal Ann. Geophys.

Discussion started: 9 May 2019

(c) Author(s) 2019. CC BY 4.0 License.

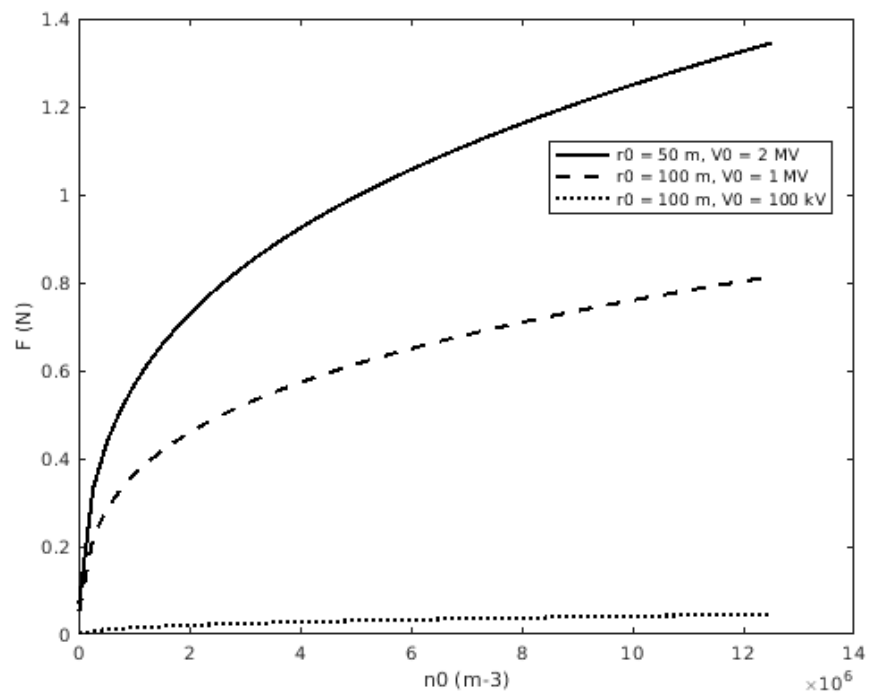

Figure 3(b): Figure 3(b) The relation between thrust $F$ and the plasma density of solar wind $n_{0}$. Dotted: $r_{0}=50 \mathrm{~m}, \boldsymbol{V}_{\mathbf{0}}=\mathbf{2} \mathrm{MV}$, dashed: $r_{0}=100 \mathrm{~m}, V_{0}=1 \mathrm{MV}$, solid: $r_{0}=100 \mathrm{~m}, V_{0}=100 \mathrm{kV}$.

Table1: scaling relation between thrust $F$ and $V_{0}, n_{0}, r_{0}$.

\begin{tabular}{cccc}
\hline$n_{0} / 10^{5} \mathrm{~m}^{-3}$ & $V_{0} / \mathrm{V}$ & $r_{0} / \mathrm{m}$ & Scaling \\
\hline 5.0 & - & 10 & $F \propto V_{0}{ }^{1.4715}$ \\
5.0 & - & 50 & $F \propto V_{0}{ }^{1.3257}$ \\
5.0 & - & 100 & $F \propto V_{0}{ }^{1.2771}$ \\
1.0 & - & 100 & $F \propto V_{0}{ }^{1.3290}$ \\
- & $100 \mathrm{k}$ & 10 & $F \propto n_{0}{ }^{0.5723}$ \\
- & $100 \mathrm{k}$ & 100 & $F \propto n_{0}{ }^{0.4360}$ \\
- & $1.0 \mathrm{M}$ & 10 & $F \propto n_{0}{ }^{0.5330}$ \\
- & $1.0 \mathrm{M}$ & 50 & $F \propto n_{0}{ }^{0.3724}$ \\
- & $1.0 \mathrm{M}$ & 100 & $F \propto n_{0}{ }^{0.3183}$ \\
5.0 & $100 \mathrm{k}$ & - & $F \propto r_{0}{ }^{0.8603}$ \\
1.0 & $1.0 \mathrm{M}$ & - & $F \propto r_{0}{ }^{0.6804}$ \\
5.0 & $1.0 \mathrm{M}$ & - & $F \propto r_{0}{ }^{0.6804}$
\end{tabular}

To estimate the electrons flux, we use the Orbital Motion Limited (OML) theory ${ }^{[1][10]}$. An electron will be captured when its trajectory meets the surface of the sphere. The electrons from solar wind coming from 1 direction, if the impact parameter (distance of electron from centre of sphere and perpendicular to its own velocity) lies within certain value, the 
Ann. Geophys. Discuss., https://doi.org/10.5194/angeo-2019-41

Manuscript under review for journal Ann. Geophys.

Discussion started: 9 May 2019

(c) Author(s) 2019. CC BY 4.0 License.

101 electron will be absorbed by the sphere. Assuming that the electrons only come from solar wind, and experience a central force.

102 With simple mechanic invariants, we can calculate the current:

$103 \quad I=\pi r_{s}^{2} e n_{0} v$,

104 Where $n_{0}$ is the density of the electron in solar wind, $v$ is the velocity of incoming electron. $r_{s}=r_{0} \sqrt{1+\frac{2 e V_{0}}{m v^{2}}}$ is the impact 105 parameter. All electrons aimed at impact parameter less than $r_{s}$ will hit on the surface of the sphere.

\section{2D Simulation}

107 In this section, we provide a PIC 2D simulation describe the behavior of the particles. The simulation region is has a cylindrical 108 symmetry along the $\mathrm{x}$-axis. Similar to figure 2, both the protons and electrons constantly flow in from the $+\mathrm{x}$ direction. The 109 electric field is solved by Coulomb equation. The potential of the sphere (spacecraft) is $V_{0}$. All the electrons moving inside the 110 sphere are absorbed.

The simulation span across a region of $18 \mathrm{~km} * 9 \mathrm{~km}$, with spatial grid varying linearly from $10 \mathrm{~m}$ to $1800 \mathrm{~m}$. The 112 grid size is small at the origin to provide a more precise result around the spacecraft. With plasma density of $n_{0}=5.0 \times$ $11310^{4} \mathrm{~m}^{-3}$, the Langmuir frequency for electron is equivalent to $\omega_{p e}=1.3 \times 10^{4} \mathrm{~s}^{-1}$. The timestep is set to $\Delta t=7.75 \times$ $11410^{-5} \mathrm{~s}$. We run 2 cases, (1) $V_{0}=1 \mathrm{MV}, r_{0}=100 \mathrm{~m}$, (2) $V_{0}=2 \mathrm{MV}, r_{0}=50 \mathrm{~m}$, with $T=32000 \Delta t$.

Figure 4 shows the established stable contour plot of the potential. We can see there is a high potential area on the incoming side of the solar wind. This means the spacecraft can couple or interact with a large range of the ambient plasma.

117 Figure 4(b) is for ion density. The concentration of ions on the right side is because the ions have nowhere to go. Figure 4(c) is for electron density; note the high concentration of electrons around the spacecraft. 
Ann. Geophys. Discuss., https://doi.org/10.5194/angeo-2019-41

Manuscript under review for journal Ann. Geophys.

Discussion started: 9 May 2019

(c) Author(s) 2019. CC BY 4.0 License.
Annales

Geophysicae

Discussions

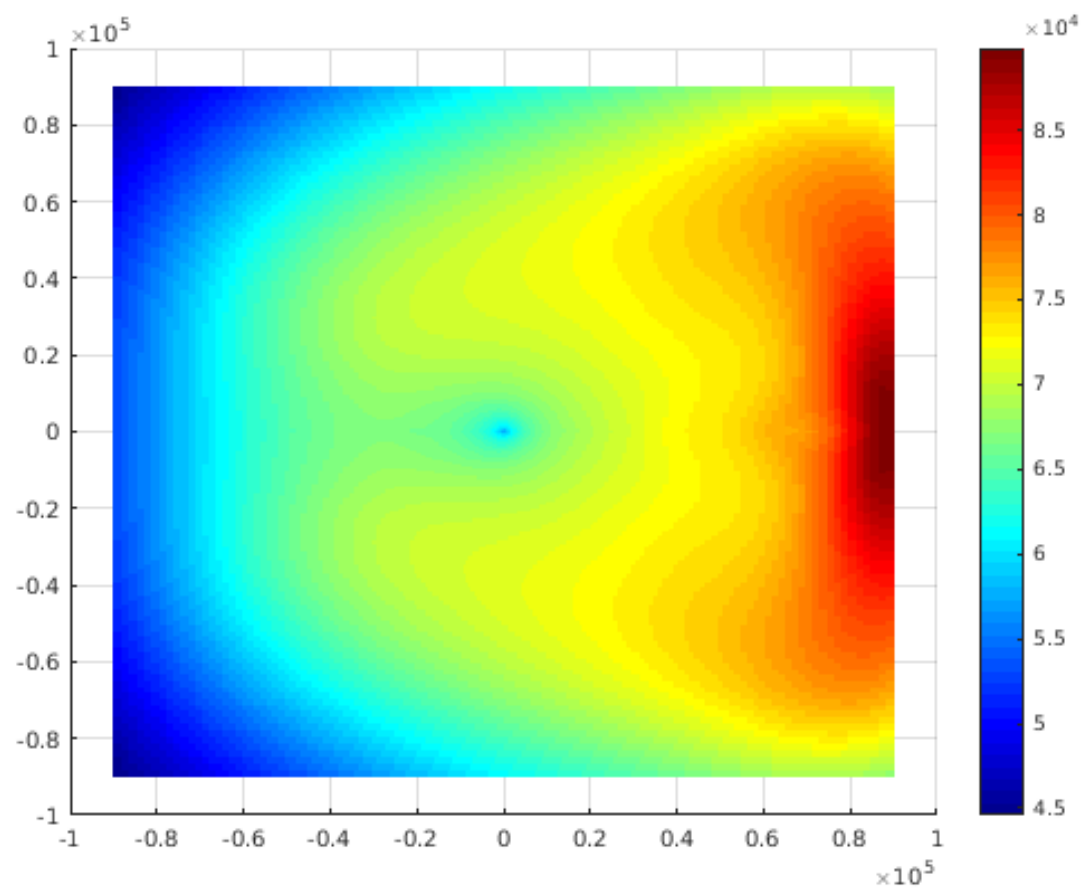

Figure 4(a): The plasma potential when simulation reached static state $\left(T=32000 \Delta t, \Delta t=7.75 \times 10^{-5} \mathrm{~s}\right)$, the spacecraft located at the origin.

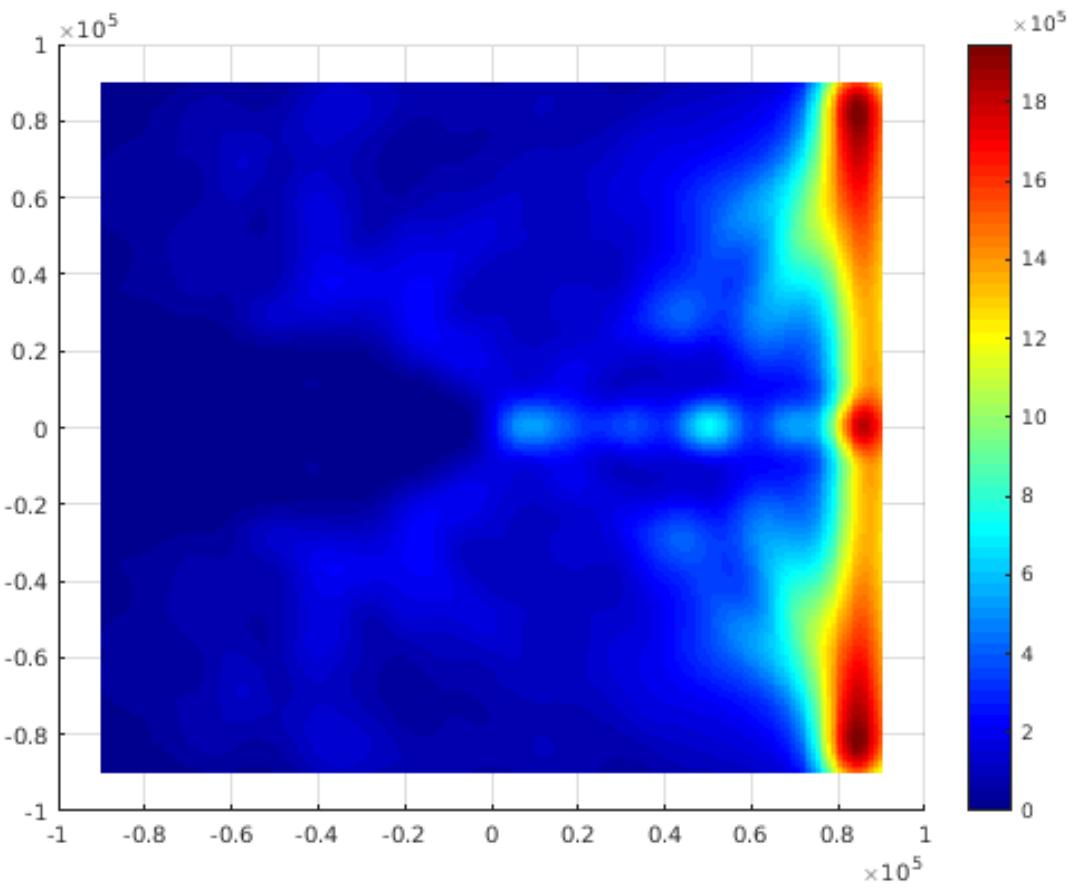


Ann. Geophys. Discuss., https://doi.org/10.5194/angeo-2019-41

Manuscript under review for journal Ann. Geophys.

Discussion started: 9 May 2019

(C) Author(s) 2019. CC BY 4.0 License.

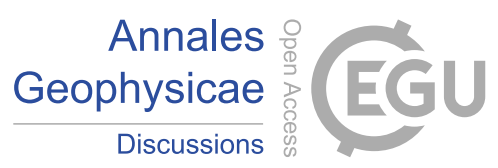

Figure 4(b): The ion density when simulation reached static state. Ions is repelled by the sphere, hence low ion density at the left side. A large portion of ions accumulation at the right side of the region is due to the boundary effect.

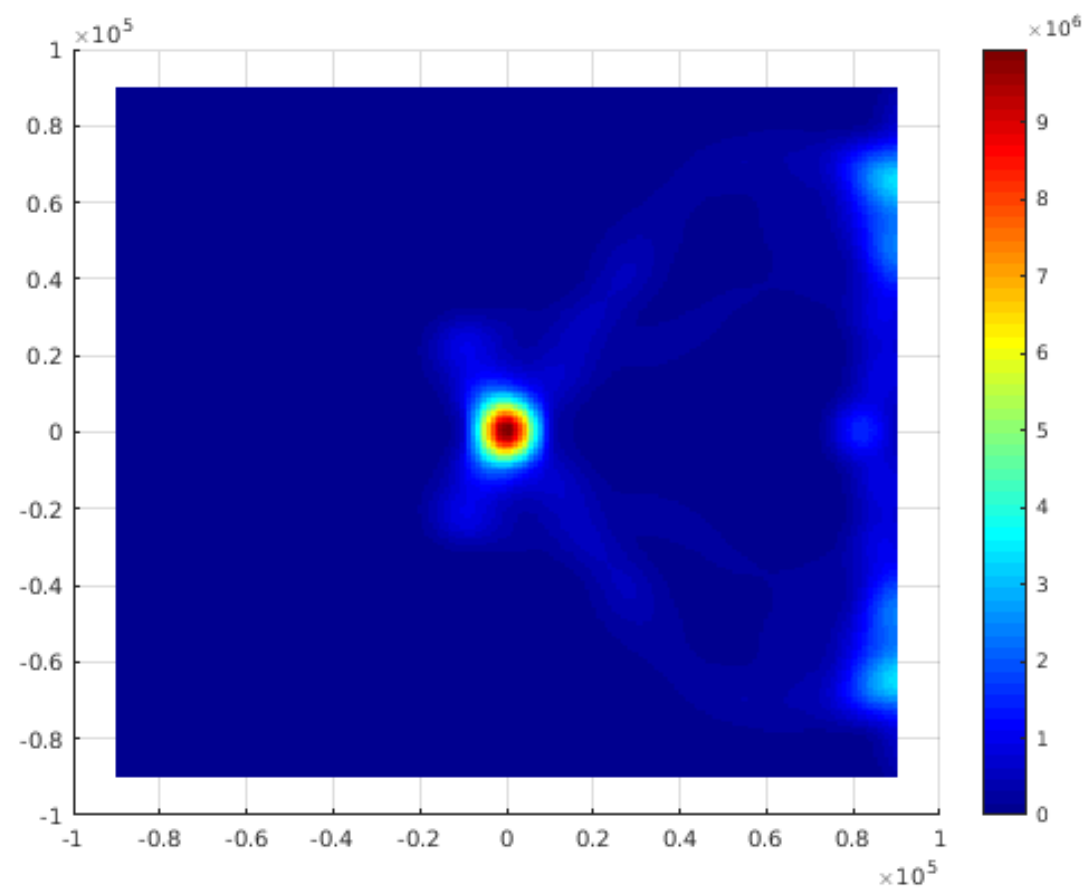

Figure 4(b): The electron density when simulation reached static state. Electrons in the region almost depleted.

The thrust acting on the sphere and the electron current is calculated and compared with 1D simulation in table 2. In 2D simulation, the thrust is 2 3 times larger than that of 1D simulation, and the electron current lower. Hence, the momentum coupling between the spacecraft and the ambient plasma is stronger than plasma shielding.

Table 2: Comparison between 1D and 2D simulation result.

\begin{tabular}{cccc|cc}
\hline \multirow{2}{*}{$V_{0}, \quad r_{0}$} & \multicolumn{2}{c}{ 1D Simulation } & \multicolumn{2}{c}{ 2D Simulation } \\
\cline { 2 - 5 } & Thrust / & Current / mA & Thrust / N & Current / mA \\
\hline $1 \mathrm{MV}, \quad 100 \mathrm{~m}$ & 0.52 & 168 & 1.6 & 102 \\
$2 \mathrm{MV}, \quad 50 \mathrm{~m}$ & 0.77 & 84 & 1.6 & 73 \\
\hline
\end{tabular}

\section{Interplanetary Travel}

133 In this section, we consider a simplified journey starting from Earth's orbit to outer planet such as Mars and Jupiter. A 134 comparison between result from 1D and 2D simulation showed that the 1D still a good approximation for thrust calculation. 
Ann. Geophys. Discuss., https://doi.org/10.5194/angeo-2019-41

Manuscript under review for journal Ann. Geophys.

Discussion started: 9 May 2019

(c) Author(s) 2019. CC BY 4.0 License.

The thrust generated depends on the ambient plasma density $n_{0}$, and $n_{0}$ drops by inversely square law of the distance, $D$, from

136 the Sun.

137 Assuming

$138 \quad F(D)=A \cdot D^{k}$,

Where $\mathrm{F}$ is the thrust, $\mathrm{D}$ is the distance to the Sun. $k$ is a scaling coefficient obtained from $1 \mathrm{D}$ simulation. $A$ is a constant determined by 2D simulation. From 1D simulation, $k$ is within the range from -1.3 to -1.5 . Adding the force into the 141 equation of motion of the spacecraft:

$142 m\left(\ddot{D}-D \dot{\theta}^{2}\right)=-\frac{G M_{s} m}{D^{2}}+F(D)$,

143 Where $M_{S}$ and $m$ are the mass of the Sun and spacecraft respectively, $\theta$ is the angular coordinate of the spacecraft in 144 a polar coordinate system with the Sun located at the center. By solving the equation of motion numerically with initial distance 145 being the radius of the orbit of the Earth, radial velocity being zero and tangential velocity equal to that of Earth, we can 146 calculate the trajectories of the spacecraft. Table 3 shows the time taken for each mission under various circumstances. The 147 main power consumption is from the electron accelerator (gun).

Table 3: Time taken for spacecraft to a journey to reach different planets.

\begin{tabular}{ccccccccc}
\hline & & \multicolumn{9}{c}{ Initial } & \multicolumn{5}{c}{ Time taken (days) } & & Power \\
$V_{0}$ & $r_{0}$ & $m$ & acceleration & & & & \\
$(\mathrm{MV})$ & $(\mathrm{m})$ & $(\mathrm{kg})$ & $\left(\mathrm{mms}^{-2}\right)$ & Mars & Jupiter & Saturn & Pluto & $(\mathrm{kW})$ \\
\hline 0.1 & 100 & 59 & 0.85 & 267 & - & - & - & 11 \\
1 & 100 & 500 & 3.64 & 80 & 290 & 446 & 1055 & 102 \\
1 & 100 & 1000 & 1.82 & 120 & 503 & 774 & 1758 & 102 \\
2 & 50 & 500 & 4.02 & 76 & 277 & 430 & 1039 & 73 \\
2 & 50 & 1000 & 2.01 & 114 & 471 & 732 & 1717 & 73 \\
\hline
\end{tabular}

The most efficient way for sending spacecraft to a target planet is by orbit transfer [11]. The spacecraft is transfer

\section{Result and discussions}


Ann. Geophys. Discuss., https://doi.org/10.5194/angeo-2019-41

Manuscript under review for journal Ann. Geophys.

Discussion started: 9 May 2019

(c) Author(s) 2019. CC BY 4.0 License.

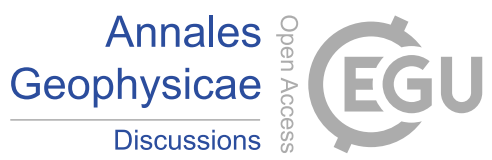

We proposed an ambient plasma momentum coupling spacecraft propelling scheme by utilizing high electric potential for the spacecraft to interact with a wide range of background plasma, which can be considered as a compact electric sail with a much smaller structure.

Preliminary calculations show that it is promising in space exploration. There are some discrepancies between the 1D and 2D simulation but generally, the order of the thrust and electron current are the same. The 2D simulation result shows that with a surface potential of the spacecraft of $1 \mathrm{MV}$, this scheme can produce a thrust of about $1.6 \mathrm{~N}$, by consuming a power of about $100 \mathrm{~kW}$. The thrust-energy efficiency is comparable to contemporary plasma thrusters (Mason et al. 2001, Richard 2004). The simulation is performed in low plasma density. In reality, this scheme could generate a larger thrust than our calculation. Since this scheme requires no propellant, it has an advantage over chemical rockets and ion thrusters. Comparing with the Esail proposed by P. Janhunen, our spacecraft require a relatively small structure (100 m versus $10 \mathrm{~km})$. It is much easier to control or manoeuver. A simple calculation shows that a spacecraft of $500 \mathrm{~kg}$ can reach Mars in 80 days and Pluto in 3 years.

The scheme is also applicable in braking, as long as the momentum of ambient plasma is exploitable. In situations like braking near Jupiter, travelling to the inner planets, the plasma trapped by Jupiter and the solar wind can be used for braking.

This scheme can achieve very high interstellar travelling speed by delivering artificial dense and energetic beams to the spacecraft over a very long distance, by, for example, a series of powerful particle accelerators on the Moon, other satellites, or dwarf planets, thus drastically shorten the travelling time to the nearest stars from tens of thousands of years to a few hundreds of years.

If the situation is favorable, such as in a cosmic jet, a spacecraft could be accelerated to relativistic velocities.

\section{References}

Bleum, H. et al.: Electron Injectors for Next Generation X-Ray Sources, SPIE 49 ${ }^{\text {th }}$ Arirzual Mtg., Denver, CO, 2-6 August (2004).

Forbes, R.G.: Extraction of Experimental ZERO-Q Evaporation Field Values, 15th Annual User Workshop of University of Surrey Ion Beam Centre, Guildford, UK, 2006, 545.

Janhunen, P.: Electric sail for spacecraft propulsion, Journal of Propulsion and Power, 20(4), 763-764, 2004.

Janhunen, P.: Coulomb drag devices: electric solar wind sail propulsion and ionospheric deorbiting, Space Propulsion 2014, Köln, Germany, May 19-22, 2014b, available at: http://arxiv.org/abs/1404.7430, 2014.

Janhunen, P.: Boltzmann electron PIC simulation of the E-sail effect, Ann. Geophys., 33, 1507-1512, 2015.

Janhunen, P. and Sandroos A.: Simulation study of solar wind push on a charged wire: basis of solar wind electric sail propulsion, Ann. Geophys., 25, 755-767, 2007.

Janhunen, P. and Toivanen P.: Safety criteria for flying E-sail through solar eclipse, Acta Astronautica, available at: http://arXiv:1502.04557, 2015. 
Ann. Geophys. Discuss., https://doi.org/10.5194/angeo-2019-41

Manuscript under review for journal Ann. Geophys.

Discussion started: 9 May 2019

(c) Author(s) 2019. CC BY 4.0 License.

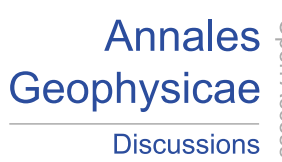

(c) (1)

187 Janhunen, P., et al.: Electric solar wind sail: towards test missions, Rev. Sci. Instrum., 81, 111301, 2010.

188 Janhunen, P. et al.: Electric solar wind sail applications overview, available at: https://arxiv.org/abs/1604.08414, 2014.

189 Janhunen, P. et al.: CUBESAT testing of Coulomb drag propulsion, available at: https://arxiv.org/abs/1604.08414, 2016.

190 Mason, L. S. et al.: 1000 hours of testing on a 10 kilowatt hall effect thruster, 2001, American Institute of Aeronautics \& 191 Astronautics, AIAA 2001-3773, 2001.

192 Richard, R. H., Development and characterization of high-efficiency, high-specific impulse xenon hall thrusters, NASA/CR— 193 2004-213099., 2004

194 Tang, X.Z., Delzanno G.L.: Orbital-motion-limited theory of dust charging and plasma response, available at: 195 https://arxiv.org/abs/1503.07820, 2015

196 Zurlev, D. N. et al.: Field ion emission: the effect of electrostatic field energy on the prediction of evaporation field and 197 charge state, J. Phys. D: Appl. Phys. 36 (2003) L74-L78, 2003. 